\title{
A Configurable Real-Time Auto-locked Software for Electronics Company (RT-ASEC)
}

\author{
Simon Salvador E. Tidon ${ }^{1 *}$, Benilda Eleonor V. Comendador ${ }^{2}$ \\ ${ }^{1}$ Software Developer of Emerson FRSI, College Instructor of Polytechnic University of the Philippines, Brgy. \\ Nanguma Junction, Mabitac Laguna Philippines, Filipino. \\ 2 Polytechnic University of the Philippines, Chief of the Open University Learning Management System \\ (OU-LMS) and the Program Chair of the Master of Science in Information Technology (MSIT) of the \\ Graduate School of the Polytechnic University of the Philippines (PUP), Filipino.
}

* Corresponding author. Tel.: 09088158172; email: sset_7@yahoo.com.

Manuscript submitted April 6, 2014; accepted January 24, 2015.

doi: 10.17706/jsw.10.10.1170-1175

\begin{abstract}
The paper was conducted to assess the importance and challenges encountered in the current machine evaluation system in an electronics company as perceived by the respondents. In addition, it also sought to understand the acceptability of the new developed Real-Time Auto-locked Software for Electronics Company (RT-ASEC). The new automated machine evaluation software can detect any irregular machine performance and can improve machine efficiency. It may help to monitor and improve the company's product yield and defects. It may also decrease the Mean Time Before Failure (MTBF) of the machine because the developed software can halt the operation once it detected any degradation in the machine's performance. Moreover, it can be re-configured easily for the new setup or environment. Its sequence of operation is easy to understand and it is adjustable to different possible user's scenarios. Furthermore, it can improve the productivity of the electronics company because the RT-ASEC's system can provide timely reports for the management that will support them in decision making.
\end{abstract}

Key Words: Real-time, mean time before failure, machine evaluation system.

\section{Introduction}

Nowadays, electronics industry are placing a higher importance on data management systems and strategies for real-time transactions and reporting. For instance, the customers want to access their financial information in real time, new regulatory requirements dictate real-time reporting of liquidity, and traders want access to real -time market and risk positions. Real-time, global data updates are becoming more common, replacing systems using nightly batch jobs for updating data that are no longer competitive [1]. Applications that require real-time processing of high-volume data streams are pushing the limits of traditional data processing infrastructures. These stream-based applications include market feed processing and electronic trading on Wall Street, network and infrastructure monitoring, fraud detection, and command and control in military environments [2]. Data analysts must make sense of increasingly large data sets, sometimes with billions or more records. We present methods for interactive visualization of big data, following the principle that perceptual and interactive scalability should be limited by the chosen resolution of the visualized data, not the number of records [3]. This is a new era where the problem is no longer one of not being able to collect enough data on our manufacturing processes, but how to 
effectively use the massive volume of data we collect wherein better analysis of the data is absolutely essential [4].

Meanwhile, Philippine electronics industry is one of the most critical industries in the Philippines. Reyes-Macasaquit stated that electronics firms in the Philippines have done upgrading covering products, processes and substantial organizational innovation. [5] As such, several system developers integrate technology to expedite the processes and protect the electronics company. For instance, the Laguna Development Corporation combined patented electronics protection technology with comprehensive monitoring and analytics applications to deliver the leading Intelligent Asset Management platform. The said solutions guard expensive and sensitive electronic assets against the damaging effects of power disturbances while simultaneously giving businesses the tools they need to predict, measure, and maximize their performance across the distributed enterprise [6].

Indeed, real-time systems cover such an enormous range of applications and products that a generalization of the characteristics into a set that is applicable to each and every system is difficult. Different categories of real-time systems may exhibit the characteristics that we identify to different extents or may not even exhibit some of the characteristics at all [7]. However, with the advent of technology real time monitoring of the manufacturing equipment and process is now possible due to the increased amounts of instrumentation and networking capabilities provided by equipment vendors. However the large amounts of data that manufacturing equipment can now provide can be overwhelming for data analysis, the networking infrastructure and the data handling availability of the database [8].

Thus, in this study the authors developed a system which utilizes real time data of an electronics company. It conducts data monitoring of machines and can sense a change in the machine's log file date time which will trigger the processing of the Real-Time Auto-locked Software for Electronics Company (RT-ASEC). Furthermore, the said system may be utilized in processing the real-time data from the machine log files and evaluates machine performance for further analysis.

\section{The Proposed System}

\subsection{System Architecture}

Fig. 1 depicts the system architecture of the proposed system. The data used in the RT-ASEC is the machine's log file. It contains all the necessary information of the product that was processed by the machine. It contains Yield, Defect and Parametric data of the product. RT-ASEC processing was triggered by the new data entry in the log file. The log file is evaluated by the system and generated pass/fail judgement and reports for hourly/daily yield, defect and average parametric results. Machine Evaluation and Reports are generated Real-Time.

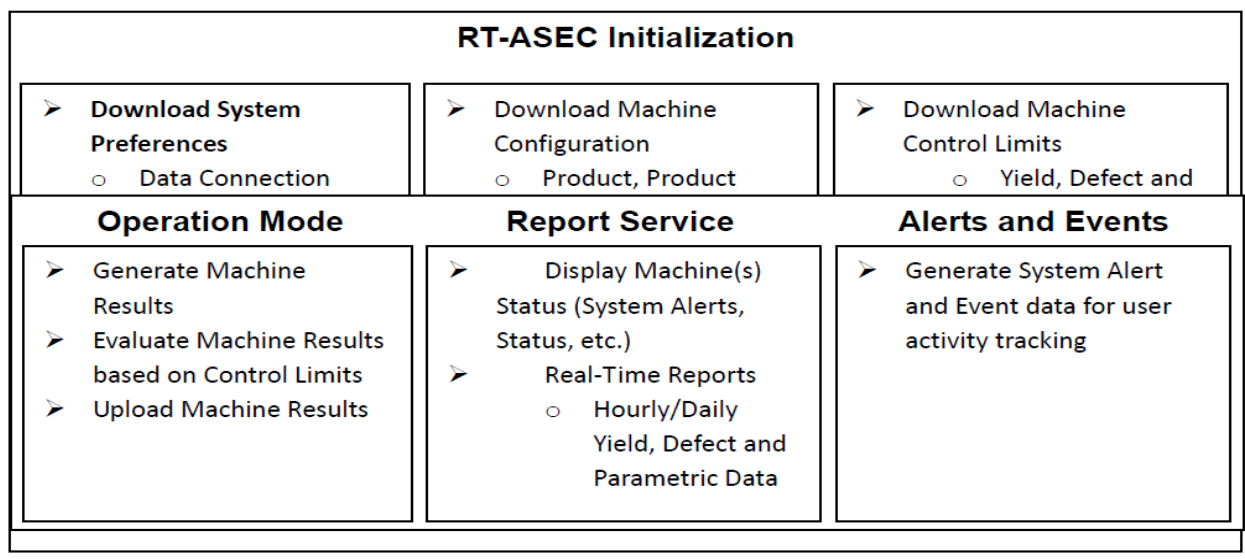

Fig. 1. Proposed system architecture. 
RT-ASEC was developed using Windows Presentation Foundation (WPF), new Technology of Microsoft for Window based application with rich Graphics support. Also RT-ASEC was developed using two Design patterns, Model-View-View Model (MVVM) and Model-View-Controller (MVC). MVVM is the design pattern that was used in Modern UI (Front-End of RT-ASEC). MVVM is the most widely used pattern for WPF. MVC is the pattern used by the application in the Business Logic area.

MySQL is the database used by the researcher in the backend area. It is an open source database system from Oracle which can support up to 10GB of data. MySQL connection string is configurable inside the RT-ASEC with a total of 27 database table to support RT-ASEC.

The 3rd party application that was used by the system was the WPF Toolkit and Modern UI. WPF Toolkit is used for the Report Service Charts and Modern UI for the Front-End of the system to have a common look and feel to the entire System UI.

\subsection{System Features}

The system has four types of users. It consists of the (1) System Administrator; (2) Engineer/Technician; (3) Operator and (4) Guests. The System Administrator has the highest right wherein RT-ASEC system Main System settings are configurable by the System Administrator only. It consists of change in System Name, Database Connection configuration, Application Control path, Company profile settings and User Management. On the other hand, Engineers/Technicians users include the Maintenance Engineers and Technicians which are capable of Product Configuration and Machine Configuration settings, Machine Log File settings, and Machine Control Limit Settings. Also Operator users are capable of accessing Operation Mode module, and Guest users which are Stakeholders (Managers, Directors), Product Engineers, and Quality assurance Engineers are capable of accessing Report Module only to view the machine reports in terms of yield, defect and parametric performance of the machine. Fig. 2 illustrates the seven (7) modules of RT-ASEC. It consists of (1) Home; (2) Product/Machine Configuration; (3) System Settings; Operation Mode; Report Service; Alerts and Events and About.

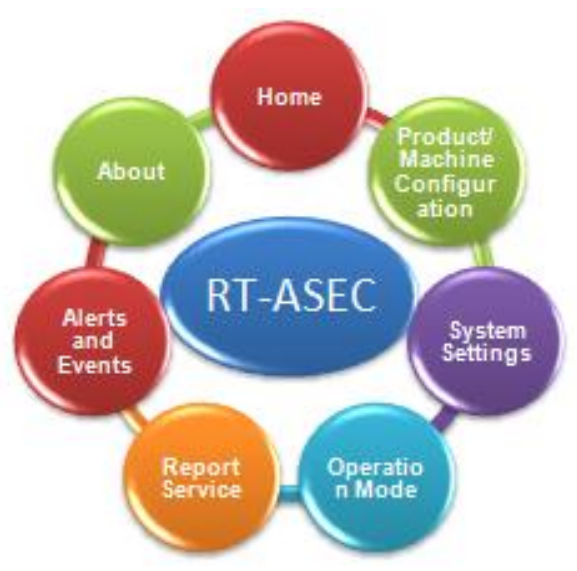

Fig. 2. RT-ASEC's modules.

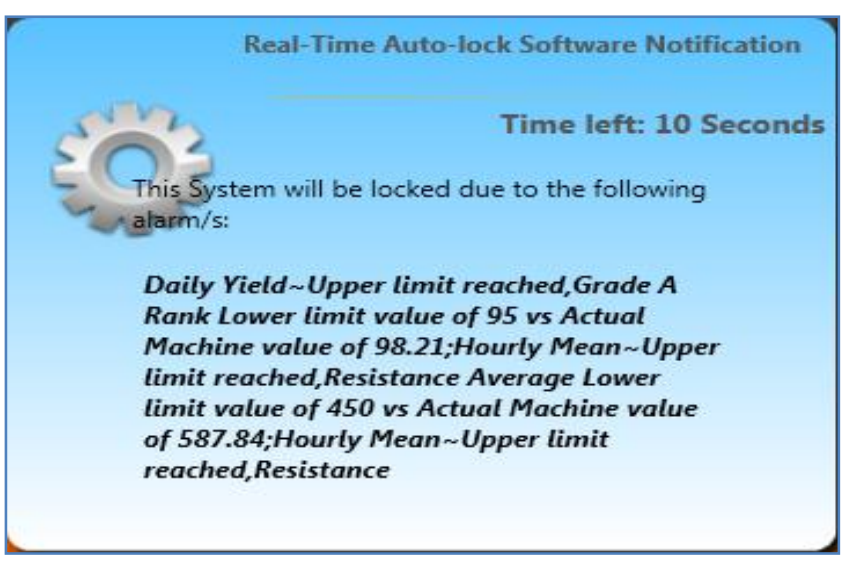

Fig. 3. RT-ASEC notification screen

Fig. 3 shows RT-ASEC has notification screen, this feature will show a notification screen prior to the lock of the machine to alarn the operator. Notification time is configurable by the System Administrator and Notification alarm is auto-generated by the RT-ASEC based on the machine control limits.

Fig. 4 shows RT-ASEC auto-locked screen, during a machine auto-locked the RT-ASEC system can only be unlcoked by the Engineers/Technician, RT-ASEC alarm is seen in the screen and the concern group can proceed to the Machine maintenance repair or activities.

Also RT-ASEC has a report feature (see Fig. 5) to view the machine performance in hourly/daily format. Machine report consists of Yield, Defect and Parametric Report of the machine as depicted in Fig.5. 
SA,Engineers/Technicians and Guest are capable of accessing the report module.

\section{RT-ASEC Auto-locked Activated}

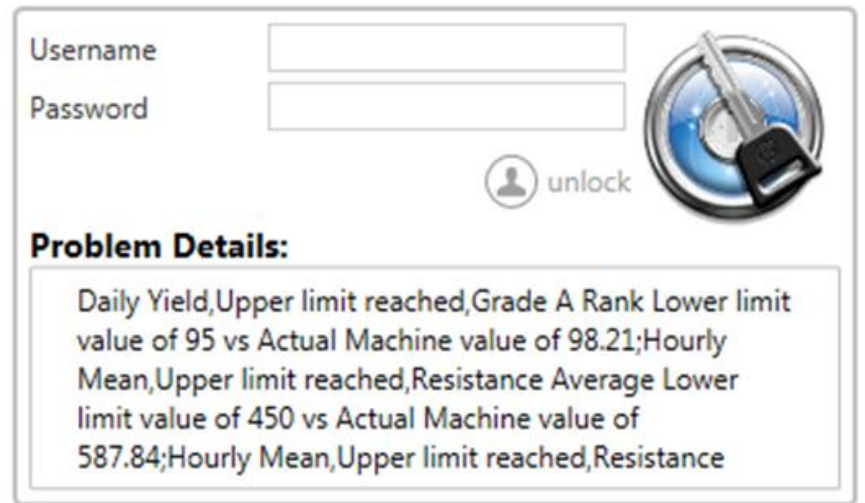

Fig. 4. Machine auto-locked screen.

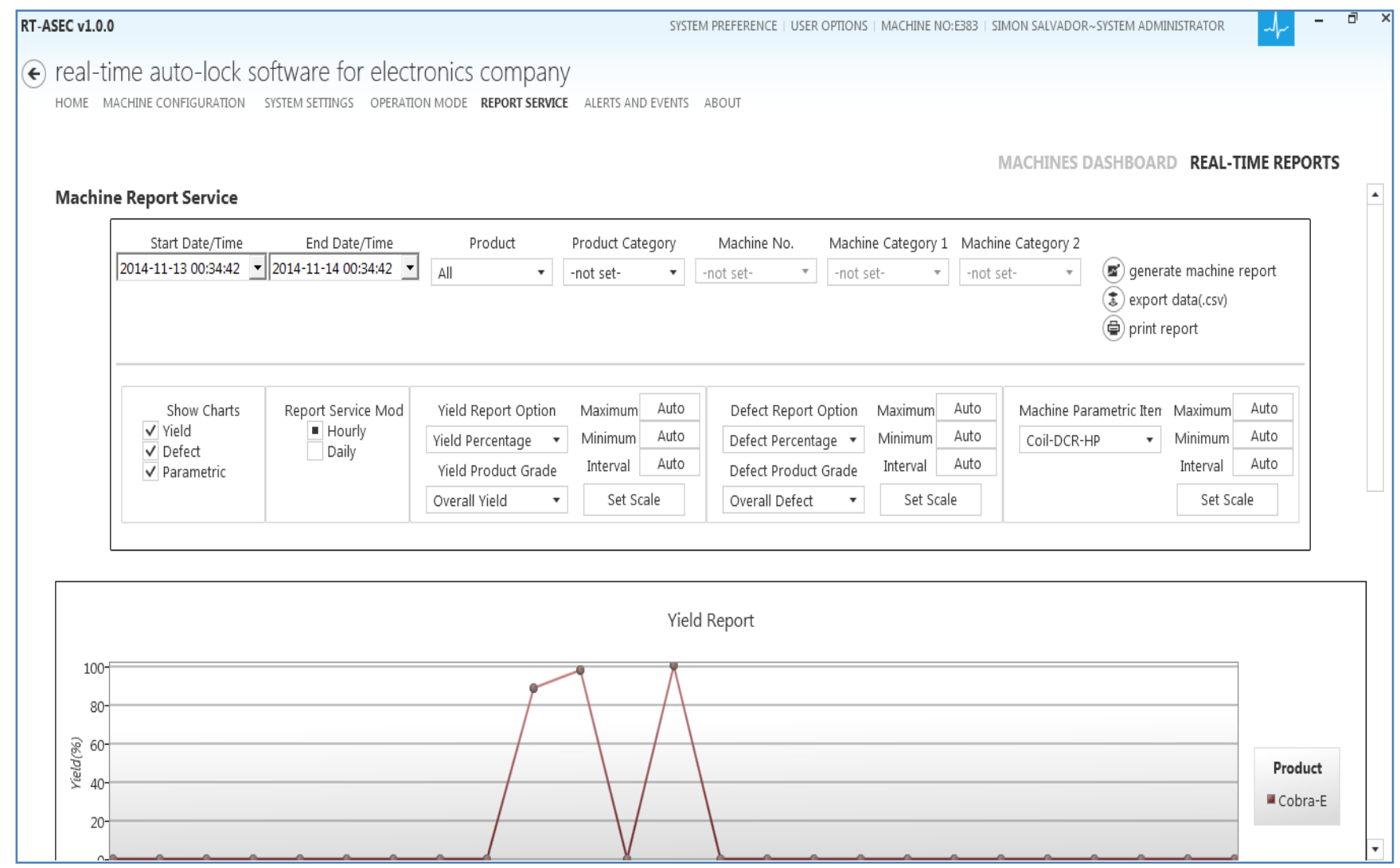

Fig. 5. RT-ASEC report module.

\section{Research Methods and Techniques}

The study explores the concepts, theories, methodologies and technologies in designing the Configurable Auto-locked software for Electronics Company. The study then presents the general requirements and issues in designing and deploying RT-ASEC's software. The authors explored the available software tools, the existing hardware and infrastructure to develop the prototype. Afterward, they collaborate with Electronics Company then conduct a developmental research. The authors gathered information that are being developed and utilized based on the current machine evaluation system. The main function of the system is to be able to evaluate a machine based on the control limits set by the technicians/engineers, 
generated a real time data, and generate Real-Time evaluations and Reports based on the machine log file.

To test the effectiveness of the developed software, a pilot test was done on February 2015. The participants were ten (10) employees in an electronics company from machine analysis technicians, machine correlation technicians, machine operators, machine maintenance technicians up to machine maintenance engineers.

\section{Results and Discussions}

The research was conducted to assess the importance and the challenges encountered in the current machine evaluation system as perceived by the respondents. In addition, it also sought to understand the acceptability of the developed software. The said system was evaluated by the respondents in terms of functionality, usability, reliability and performance.

Table I illustrates the summary of the respondents' level of acceptance of the developed system. It can be observed from the table that in terms of "Functionality" the respondents said that the system is "Highly Acceptable' which ranked first and obtained the mean response of 4.42. It is closely followed in the second rank by "Reliability" with a mean response of 4.37verbally interpreted as 'Highly Acceptable'. The third in the rank is "Performance" with a mean response of 4.35 and verbally interpreted as "Highly Acceptable". Furthermore "Usability" rank as the last which has a mean response of 4.25 and a verbally interpreted as "Highly Acceptable".

Table 1. Respondents' Level of Acceptance of the Developed System

\begin{tabular}{|c|c|c|c|}
\hline Criteria & Mean Response & Interpretation & Rank \\
\hline Usability & 4.25 & Highly Acceptable & 4 \\
\hline Functionality & 4.42 & Highly Acceptable & 1 \\
\hline Reliability & 4.37 & Highly Acceptable & 2 \\
\hline Performance & 4.35 & Highly Acceptable & 3 \\
\hline
\end{tabular}

\section{Conclusions and Future Works}

Based on the evaluation made by the respondents on the proposed system, the researchers arrived to the following conclusions: Real-Time data generation is essential in an electronics company to provide immediate machine evaluations, actions and responses. The developed automated machine evaluation system can detect irregular machine performance and it may improve machine efficiency. It also decreases the machine's Mean Time Before Failure (MTBF) because the machine can be stopped immediately whenever there is already degradation in the machine's performance. It can improve electronics company work force in terms of productivity because the RT-ASEC's system will provide the judgment for them. It may also enhance the machine efficiency which improves machine judgment with respect to the product performance. Auto-lock System is capable of locking the workstation whenever the system receives an alert with regards to the machine's performance. It also prevents the users for by-passing the system's alerts and provides a notification before locking.

Real-Time Yield, Defect and Parametric Report generation increases productivity. Users can automatically generate Real-Time charts report for machine performance analysis. Real-Time report generations are 
appropriate to the needs of the company. RT-ASEC's provides accurate results and produce multiple reports instantly. It can provide vital information for decision making of the organizational key players.

Real-Time Auto-lock Software for Electronics Company is easy and convenient to use. RT-ASEC system can also be re-configured easily for the new setup or environment. Its sequence of operation is easy to understand. The system is adjustable to different possible user scenarios. System can recover from mistakes quickly and easily and response and processing time are acceptable.

\section{References}

[1] Scott K. D. (2012), Financial Services industry Vertical, VMware Real-Time Data Management in the Financial Services Industry with VMware vFabricGemFire.

[2] Zhicheng, L., Biye J., \& Heer, J. (2013). Real-time Visual Querying of Big Data.

[3] Schreiber, P. (2012). VP sales and marketing, northwest analytical. 21st Century Process Management Using SPC Based on Manufacturing Analytics.

[4] Sandeep, S. (1996). Mechanical Engineering. Ohio State University.

[5] Macasaquit, M. (March 2010). In the Case Study of the Philippine Electronics Industry.

[6] Innovolt 2014 of Laguna Development Corporation.

[7] Mall, R. (2006). Time Systems: Theory and Practice Pearson Education India September 14, 2006.

[8] Orphanides., \& Williams, J. C. (2006). Monetary policy under imperfect knowledge. Journal of the European Economic Association, 4 (2-3), 366-375.

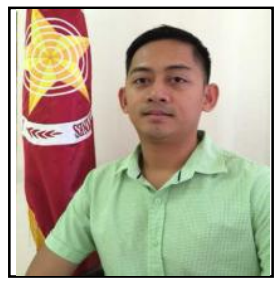

Simon Salvador E. Tidon is a member of MSIT group of the Polytechnic University of the Philippine. He earned his master of science in information technology (MSIT) from the Polytechnic University of the Philippine Graduate School in 2015. He is in the field of academe since 2011 as a college instructor 1 in the Polytechnic University of the Philippine and currently employed in emerson fisher-rosemount process management as a software developer. Mr. Tidon's field of specializations is management information system, system analysis and design and some programming languages.

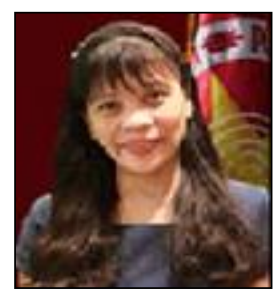

Benilda Eleonor V. Comendador was a grantee of the Japanese Grant Aid for human resource development scholarship (JDS) from April 2008 to September 2010. She obtained master of science in global information telecommunication studies (MSGITS), majoring her in project research at Waseda University, Tokyo Japan in 2010. She was commended for her exemplary performance in completing the said degree from JDS. She finished her master of science in information technology at Ateneo Information Technology Institute, Philippines in 2002. Presently, she is the chief of the Open University Learning Management System (OU-LMS) and the program chair of the master of science in information technology (MSIT) of the Graduate School of the Polytechnic University of the Philippines (PUP). She is an associate professor and was the former chairperson of the Department of Information Technology of the College of Computer Management and Information Technology of PUP. She attended various local and international computer related trainings and seminars and was the country's representative to the Project Management Course in 2005, which was sponsored by the Center for International Computerization Cooperation (CICC) in Tokyo, Japan together with other 9 representatives from various ASEAN countries. 